Cahiers de civilisation médiévale

\title{
Collectif, The Complete Story of the Grail: Chrétien de Troyes' Perceval and its Continuations
}

Jean-François Poisson-Gueffier

\section{(2) OpenEdition}

1 Journals

Édition électronique

URL : https://journals.openedition.org/ccm/5073

DOI : $10.4000 / \mathrm{ccm} .5073$

ISSN : 2119-1026

Éditeur

Centre d'études supérieures de civilisation médiévale/Université de Poitiers

Édition imprimée

Date de publication : 1 septembre 2020

Pagination : 166-169

ISBN : 978-2-490783-06-9

ISSN : 0007-9731

\section{Référence électronique}

Jean-François Poisson-Gueffier, "Collectif, The Complete Story of the Grail: Chrétien de Troyes' Perceval and its Continuations », Cahiers de civilisation médiévale [En ligne], 250-251 | 2020, mis en ligne le 01 septembre 2020, consulté le 09 décembre 2022. URL : http://journals.openedition.org/ccm/5073 ; DOl : https://doi.org/10.4000/ccm.5073

\section{(c) (i) 9}

Creative Commons - Attribution - Pas d'Utilisation Commerciale - Pas de Modification 4.0 International - CC BY-NC-ND 4.0

https://creativecommons.org/licenses/by-nc-nd/4.0/ 
C. Chazelle accorde ainsi à Bède le Vénérable la place qu'il mérite d'occuper dans la construction de l'idéal monastique du haut Moyen Âge et ses objectifs théologiques et liturgiques. Au cœur de ce processus sophistiqué, on trouve un rapport particulier au temps chrétien auquel Bède le Vénérable donne une place de premier plan. Pour le théologien anglo-saxon du $\mathrm{VIII}^{\mathrm{e}} \mathrm{s}$., l'une des clés essentielles pour permettre à l'Homme d'entrer dans la compréhension du texte biblique est la perception de l'eschatologie et du rapport de l'Homme à la temporalité de la conception eschatologique. À côté de cela, Bède focalise son attention sur l'importance accordée dans l'exégèse et la théologie de façon plus générale, au rapport entre le libre arbitre de l'Homme et la grâce divine. On doit donc à C. Chazelle la mise en relief de ces éléments essentiels pour la compréhension d'une espèce de « réforme » monastique et religieuse qui n'en porte pas le nom mais dont tous les éléments semblent réunis au monastère de Wearmouth-Jarrow à la jonction des $\mathrm{VII}^{\mathrm{e}}$ et $\mathrm{VIII}{ }^{\mathrm{e}} \mathrm{s}$.

Le chap. 3 dédié à l'examen codicologique, paléographique, textuel du codex Amiatinus et des manuscrits ou fragments qui lui sont reliés, constitue un moment fort du livre. Dans le cadre d'une érudition sans failles, C. Chazelle démontre l'existence de liens génétiques entre ces manuscrits et les fragments conservés mais aussi avec d'autres témoins majeurs de la production issue d'autres grands centres de réalisation de manuscrits bibliques ou liturgiques ailleurs qu'à Wearmouth-Jarrow, comme au monastère de Lindisfarne.
Les chap. 4 et 5 du livre sont principalement dédiés au codex Amiatinus, à l'iconographie de ses peintures et à son "message » théologico-liturgique. Ici, C. Chazelle explore avec succès les aspects liturgiques - notamment les thèmes de lecture à la messe impliqués par le Codex Amiatinus, probablement terminé en 703, et l'iconographie de peintures telle que la Maiestas Domini où le thème de la christologie est fortement affirmé et qui a fait certainement partie d'additions au manuscrit d'origine autour de 710-716. Sur tous ces sujets il faut savoir gré à l'a. d'avoir échappé à l'approche strictement artistique au profit d'une lecture et d'une interprétation prenant en compte tous les éléments codicologiques au sens large du terme du manuscrit principal du groupe étudié et ce, sans jamais rien négliger de l'historiographie des recherches sur l'enluminure de cette époque dans les îles anglo-saxonnes et sur le continent. Ces remarques peuvent être appliquées au chap. 6 qui traite du thème du cadeau fait à Saint-Pierre à Rome du codex Amiatinus accordant à nombre de peintures du manuscrit et à leur iconographie une valeur théologico-politique dépassant le seul cadre de l'illustration biblique. Enfin, le chap. 7 explore très utilement l'« afterlife » du codex Amiatinus jusqu'à aujourd'hui soulignant ainsi la force et le pouvoir de ces « objets » si particuliers que sont les précieux manuscrits peints du haut Moyen Âge.

Au final, le livre de C. Chazelle est à tous égards une grande réussite où les hypothèses et arguments, servis par une remarquable érudition, emportent l'adhésion.

Éric PALAzzo

UMR $7302-$ CESCM

Université de Poitiers

The Complete Story of the Grail: Chrétien de Troyes' Perceval and its Continuations, N. Bryant (trad.), Woodbridge, D. S. Brewer (Arthurian Studies, 82), 2018 (rééd. 2015).

L'inachèvement qui frappe l'écriture du Conte $d u$ Graal cristallise fantasme et volonté d'en porter la matière à son plus haut degré d'accomplissement; fantasme excité par la suspension du récit sur une question - «Et quant la reïne la voit / Si li demande qu'ele avoit» (« Perceval ou le Conte du Graal», dans Chrétien de Troyes, Euvres complètes, D. Poirion [dir.], A. Berthelot, P. F. Dembowski, S. Lefèvre, K. D. Uitti et P. Walter [collab.], Paris, Gallimard [Bibliothèque de la Pléiade, 408], 1994, p. 911, v. 9233-34); volonté de complétude liée au pouvoir de fascination que procurent le Graal, la figure de Perceval et un univers de fiction riche de virtualités. Dans le vaste lacis des récits arthuriens qui repensent et reconfigurent ses données élémentaires (cour arthurienne, quête, aventure, merveille...), les quatre Continuations présentent autant de fictions centrifuges qui néanmoins convergent vers une résolution, résolution atteinte par des chemins de traverse, les trois dernières Continuations se distinguant par un esprit d'indépendance croissant.

Le corpus des Continuations met en œuvre une dialectique de la continuité et de sa solution qui, par-delà une apparente dispersion, trouve son origine profonde dans « la faute originelle, la faille qui marque de 
façon indélébile, le récit de la naissance, [et qui] avait induit une économie narrative qui ne peut trouver de butée d'arrêt : de vengeance en vengeance, de corps en corps, de récit en récit, la blessure initiale migre sans fin » (Alexandre LeupIN, « La faille et l'écriture dans la première continuation du Perceval», Le Moyen Âge, 88, 1982, p. 237-269).

Les Continuations ont été antérieurement traduites en anglais, mais cette nouvelle édition présente l'intérêt de les envisager d'un seul tenant. La parution de ce volume s'inscrit dans un projet de grande ampleur dont il convient de louer l'ambition : mettre à la portée d'un public anglophone les principaux romans du corpus arthurien appartenant au domaine français. Cette édition du Conte du Graal, de ses quatre Continuations et de ses prologues (Élucidation et Bliocadran), a ainsi été précédée, également chez D. S. Brewer, de celle du Perlesvaus (The Hight Book of the Grail: a Translation of the ThirteenthCentury Romance of Perlesvaus, N. BRYANT [trad.], Cambridge, D. S. Brewer, 2007 [réed. 1978]), de la trilogie attribuée à Robert de Boron (Merlin and the Grail: Joseph of the Arimathea, Merlin, Perceval: the Trilogy of Proses Romances attributed to Robert de Boron, N. Bryant [éd.], Cambridge, D. S Brewer [Arthurian Studies, 48], 2001), d'une édition complète (The Complete Story of the Grail: Chrétien de Troyes Perceval and its Continuations, N. Bryant [trad.], Cambridge, D. S. Brewer, 2015) puis d'une anthologie du Perceforest (Perceforest: The Prehistory of King Arthur's Britain, N. BRYANT [trad.], Cambridge, D. S. Brewer [Arthurian studies, 77], 2011). Producteur à la BBC, Nigel Bryant semble ainsi animé d'un inextinguible désir de promotion des lettres médiévales.

Gage indéniable de scrupule philologique, la traduction en anglais repose sur l'édition du Conte $d u$ Graal (Le roman de Perceval ou le conte du Graal, W. RoAch [éd.], Genève/Paris/Droz, Librairie Minard, 1959 [2éd.]) établie par William Roach et unanimement saluée à sa parution, sur les vol. II, IV et $\mathrm{V}$ des Continuations of the Old French "Perceval" of Chretien de Troyes (Philadelphie, The American Philosophical Society, 1965-1983) et, pour la seule Continuation de Gerbert de Montreuil, sur l'édition de Mary Williams (Gilbert de Montreuil, La continuation de Perceval, M. Williams [éd.], Paris, Champion [Les Classiques français du Moyen Âge, $28,50], 1922)$. Le titre de ce volume, réédition de la version publiée en 2015, s'avère quelque peu déceptif. « The Complete Story of the Grail » induit un double principe d'unicité que démentent les multiples « mutations cosmogoniques » qui président aux réécritures du mythe arthurien (Patrick MoRAN, «Conter ou commémorer : la temporalité arthurienne dans le Cycle Vulgate et dans le Morte Darthur, autour de deux épisodes de la vie de Lancelot », Temps et mémoire dans la littérature arthurienne : actes du colloque international de la branche roumaine de la société internationale arthurienne (Bucarest, 14-15 mai 2010), C. GîrbeA, A. Popescu et M. Voicu [dir.], Bucarest, Editura Universitatii din Bucureşti [Mediaevalia, 2], 2011, p. 389-399). Le Conte du Graal et ses Continuations ne forment, à proprement parler, qu'une " histoire du Graal », " complète » mais non exhaustive - car nulle ne saurait l'être -, et marquée par de très nombreuses lignes de faille.

L'introduction (XVIII-LI) énonce à la manière d'un axiome la relation de l'« incomplete poem » et de ses Continuations, à travers trois opérations complémentaires («taken up, expanded and finally brought to a conclusion », XVIII) envisagées en un flux narratif continu, alors que seuls deux manuscrits sur les quinze témoins conservés comportent l'ensemble des Continuations. Chaque œuvre est succinctement résumée, mais leur signification abordée de manière superficielle - les pages consacrées au Conte du Graal considèrent d'un style cursif l'ironie de Chrétien et la dimension symbolique de certains actes, délaissant un vaste empan d'enjeux poétiques et esthétiques. La transition qui articule la présentation du Conte à celle de ses Continuations se place ainsi très en deçà des incidences inhérentes à cette suture narrative : "but the question is: did his continuators see and sense it » (XXII), « it » désignant l'ironie de Chrétien à l'égard du monde chevaleresque.

Le résumé des Continuations se polarise principalement sur des objets - le Graal et l'épée brisée - et la valeur narrative et symbolique attribuée aux aventures (vindicatives dans la Continuation Manessier) et aux personnages (Perceval figuré en nice dans le Conte du Graal, en alter Christus dans la Continuation de Gerbert de Montreuil...). Les vertus de ce résumé sont nombreuses : dénouer les principaux fils de l'intrigue, en faire saillir les arêtes, donner un aperçu des conditions qui ont historiquement présidé à l'écriture de chaque continuation («sequel »), comme de chaque avant-texte («prequel »). L'édition comporte ainsi en appendice l'Élucidation et Bliocadran, dont le traducteur ne cèle rien de la part d'ombre et de mystère, tant pour ce qui concerne le lexique et la syntaxe (Élucidation) que la narration et l'appartenance à un cycle, existant ou perdu. Le tour d'horizon des œuvres traduites dans ce volume s'achève par quelques considérations sur l'oralité ( Reading aloud ») et son influence sur l'interprétation du récit - dans toutes 
les acceptions que recouvre le mot. La pensée de Paul Zumthor infuse ces pages, sans être explicitement invoquée ni atteindre un degré comparable de conceptualisation.

Les aspects matériels de l'édition - mise en page, ventilation des épisodes et séquences, notes explicatives de bas de page - en favorisent assurément la lecture. La table des matières (V-XV) donne une image de la succession des aventures à travers un titre de portée générale, précisé par des subdivisions qui en détaillent les phases. Les précisions fournies par le glossaire (qui rappelle en italique la nature, la fonction et, le cas échéant, l'origine de personnages étrangers au Conte et à ses Continuations) dénotent une édition des plus soignées.

Que l'apport de la critique n'ait pas été mis à profit, pour des élucidations ponctuelles comme pour des interprétations d'ensemble constitue nonobstant la principale lacune de cette Complete Story of the Grail, à laquelle manque une véritable profondeur de champ. La mise à disposition du texte aurait gagné, pour un résultat pleinement efficient, à s'enrichir d'une démarche de vulgarisation scientifique qui, même en l'absence de vocabulaire savant et de lectures érudites, aurait donné tout son sens à l'inscription de ce volume dans la prestigieuse collection des «Arthurian Studies » de D. S. Brewer.

En ce sens, les rares débats sur des questions d'interprétation ne révèlent pas une posture convaincante, se fondant sur des assises méthodologiques friables et portant sur des aspects parfois mineurs, qui n'approfondissent en rien la compréhension des textes. Ainsi lorsque N. Bryant bat en brèche W. Roach sur l'appartenance, pour les médiévistes, de la Première Continuation au monde du Conte $d u$ Graal, introduisant des réserves péremptoires («this is missing the point »), celles-ci ne présentent pas un intérêt littéraire de première force, de surcroît en l'absence de toute démonstration (XXIII). Non seulement les références synoptiques - en langue anglaise, Leah Tether, The Continuations of Chrétien's Perceval: Content and Construction, Extension and Ending, Cambridge, D. S. Brewer (Arthurian Studies, 79), 2012 et Thomas Hinton, The Conte Du Graal Cycle: Chrétien de Troyes' Perceval, the Continuations and French Arthurian Romance, Cambridge, D. S. Brewer (Gallica, 23), 2012 - font défaut, mais le traducteur élit des angles d'analyse qui délaissent les questions les plus essentielles pour l'élucidation du sens. La christianisation progressive du Graal et la présence de la violence sont ainsi éludées.
Au rang des imprécisions ou traces d'ingénuité, doivent être relevées les épithètes accordées à Perceval («Everyman » devenu «Everyknight» et «Everyyouth», ce qui ne va pas sans quelque contresens) ou la surprise éprouvée par N. Bryant face au rôle dévolu au roi Arthur dans la Première Continuation : " and what a behaviour of an ideal king? King Arthur in these stories does very little ». À cet égard, les Continuations ne sont ni le Conte du Papegau ni la première branche du Perlesvaus. De même, lire dans les métamorphoses du Graal au sein de la Première Continuation une marque de l'insignifiance qui lui est infligée aurait grandement bénéficié de l'approche proposée par Annie Bertin et Annie Combes, qui étudient les subtilités de la réécriture d'un « conteur » qui a « substitué au merveilleux elliptique de Chrétien un surnaturel angoissant » (Écritures du Graal, Paris, Presses universitaires de France [Recto-Verso], 2001, p. 42). De cette étude, l'a. aurait fructueusement retiré les critères de définition d'une continuation : un « raccord imperceptible » et l'inscription dans un « texte à contraintes », le développement de « situations restées à l'état d'amorce », une « symbiose tendancielle » marquée par « la reprise de séquences textuelles soumises aux procédures de reformulation » (ibid, p. 32).

D'autres approches de cette tectonique narrative ont conféré à l'ensemble une signification unitaire, à l'image de la thèse remarquable de Sébastien Douchet, qui montre que « le continu se crée à partir du discontinu » et que le lien sémantique qui unit continuation et continuité est de pure corrélation : « intrinsèquement fondée par un pacte de continuation, l'écriture de nos romans ne s'invente qu'au prix de stratégies qui nouent, dénouent et renouent les liens qui les unissent avec des hypotextes qu'ils prolongent ad libitum, défaisant presque la notion de fin, c'est-à-dire d'achèvement et de finalité du roman » (Sébastien Douchet, Logiques du continu et du discontinu : espace, corps et écriture romanesque dans les Continuations du Conte du Graal (11901240), thèse inédite de l'Université Paris-Sorbonne, dirigée par Dominique Boutet, 2004, p. 477).

Si l'un des comptes rendus des Faits et Conquêtes d'Alexandre le Grand de Jehan Wauquelin (The Medieval Romance of Alexander: Jehan Wauquelin's The Deeds and Conquests of Alexander The Great, N. BRYANT [trad.], Woodbridge, The Boydell Press, 2012), reconnaissait tout uniment la qualité d'un massif qui, à l'instar de ce volume, représente un investissement considérable, cette nouvelle publication échoue à « toucher un public 
varié d'étudiants et de chercheurs provenant de toutes les disciplines » (Jessica Stoll, « Nigel Bryant, The Medieval Romance of Alexander », Cahiers de recherches médiévales et humanistes, 2012, en ligne : http://journals.openedition.org/crm/13045). L'écriture fluide, animée, empreinte d'exaltation et de fièvre, qui restitue aux romans toute leur vigueur, est tout entière emportée dans un flux d'aventures placé sous le signe du seul plaisir que procure le texte. Une introduction et des notes plus substantielles - et non forcément d'une longueur accrue - auraient alors prodigué bien des ressources pour appréhender cette vaste fresque. L'a. n'en possède pas moins une connaissance intime des récits arthuriens, dont semble ne subsister pour lui aucune terra incognita. À force de feuilleter ces pages de main nocturne et journelle, il s'est acquis une science arthurienne qui force l'admiration.

La langue que déploie le traducteur est à cet égard d'une grande précision et s'applique à cerner les nuances du texte médiéval. « Significance », préféré à «meaning » pour rendre l'idée de senefiance (p. 359), constitue, entre mille autres exemples, la marque d'une attention portée au mot juste, et préserve en un même geste le lissé propre à toute traduction empreinte d'élégance.
En vue de conférer aux récits arthuriens une illusoire «potent, timeless resonance 》 (XIX) parmi les lecteurs de notre temps, l'a. multiplie parallèles et incitations à retrouver en soi et en sa vie les signes que disséminent les aventures chevaleresques. Cette pratique anglo-saxonne de la littérature, alternative à la codicologie et à l'herméneutique privilégiées dans les universités de langue française, ne manque pas d'être déroutante, mais semble fructueuse si l'on considère les fins qu'elle s'assigne : non tant pénétrer les arcanes d'une poétique du roman arthurien, que tendre vers la seule jouissance esthétique d'un récit aux épisodes « richly entertaining » (XXIV).

Les éléments de bibliographie ( Further Readings», LI-LII), sont emblématiques, par leur ténuité et leur défaut d'ouverture à la recherche non anglo-saxonne, de la destination d'une somme digne de tous les éloges dans le cadre d'une lecture d'agrément, mais qui s'avère infiniment trop lacunaire pour servir la recherche.

Jean-François PoIsSON-GuefFiER Lycée Pierre d'Ailly de Compiègne

Las fronteras pirenaicas en la Edad Media (siglos VI-XV) / Les frontières pyrénéennes au Moyen Âge (VI$X V^{e}$ siècles), Sébastien Gasc, Philippe Sénac, Clément Venco, Carlos Laliena (éd.), Saragosse, Prensas de la Universidad de Zaragoza, 2018

Cet ouvrage est un bon apport au sujet des rapports entre les deux versants des Pyrénées au Moyen Âge. Il répond à des caractéristiques précises qui encadrent et expliquent les 15 chapitres, outre l'introduction et les conclusions, qui le composent. En premier lieu, il est le fruit d'une rencontre organisée en 2011 par le laboratoire Framespa de l'Université de Toulouse II Jean Jaurès (alors, et jusqu'en 2014, Toulouse II-Le Mirail) en collaboration avec le groupe de recherche en études médiévales de l'Université de Saragosse (Cema) et en étroit contact avec le groupe Ocorde composé d'un secteur de médiévistes de l'Université autonome de Barcelone. En deuxième lieu, comme indiqué dans la préface, il s'agissait de rassembler de jeunes auteurs, soit en train de réaliser leur thèse de doctorat soit l'ayant terminée, avec la conviction, ajoutée dans le titre du colloque, de faire équivaloir « nouvelles thèses, nouveaux débats ».

Cela apporte une forte cohérence aux paradigmes explicatifs et aux emplois bibliographiques du livre. Une participation plus plurielle quant aux provenances et l'inclusion de quelques auteurs ayant davantage de maturité (maturité, non pas dans le sens de corriger ce qu'on affirme maintenant, mais dans celui de compléter progressivement le bagage interprétatif de base avec des perspectives non issues des enseignements initiaux) auraient pu être plus enrichissantes.

En ce qui concerne le cadre chronologique, le titre énonce un traitement entre le $\mathrm{VI}^{\mathrm{e}}$ et le $\mathrm{Xv}^{\mathrm{e}} \mathrm{s}$. Dans la pratique, 13 études s'enchaînent de façon bien ordonnée entre le $\mathrm{VI}^{\mathrm{e}}$ et le XII ${ }^{\mathrm{e}} \mathrm{s}$., auxquelles s'ajoutent, de façon plus détachée, un texte sur le XIII ${ }^{\mathrm{e}}$ et un autre sur le XIV ${ }^{\mathrm{e}} \mathrm{s}$.. Quant au cadre géographique, les textes se centrent sur les rapports entre les deux côtés des Pyrénées sauf trois, qui parlent de territoires concrets assez proches des Pyrénées : le nord-est de la Tarraconnaise, le Sobrarbe et la Ribagorce. Ces deux derniers conservent, toutefois, la référence concernant la frontière : à propos du Sobrarbe par le contact, parfois armé, avec l'espace andalou, et à propos de la Ribagorce pour encadrer l'étude dans le contexte de la révision de la délimitation entre l'Aragon et la Catalogne. 